\title{
Guidelines are too important to be left to clinical experts
}

\author{
Mattias Johansson PhD, Pär Stattin MD PhD
}

See related research article by Dinnes and colleagues on page 169 and at www.cmaj.ca/lookup/doi/10.1503/cmaj.110600

La guerre! C'est une chose trop grave pour la confier à des militaires.

(War is too important a matter to be left to the military.) — Georges Clemenceau

$\mathrm{T}$ o paraphrase the words of the late French statesman: guidelines are too important a matter to be left to clinical experts. That is the bottom line of the research article by Dinnes and colleagues ${ }^{1}$ on the extent to which clinical guidelines for monitoring prostate-specific antigen in patients after curative treatment for prostate cancer are based on the available scientific evidence.

The risk of recurrence and death from cancer varies widely in men with localized prostate cancer who have received curative treatment. This risk varies substantially depending on the extent and differentiation of the tumour, the status of the lymph nodes, the margins of the surgical specimen, in addition to the preoperative and postoperative serum levels of prostate-specific antigen. Thus, it would seem crucial that clinical guidelines with recommendations for monitoring patients whose prostate cancer has been curatively treated are based on high-quality scientific evidence.

In their recent paper, Dinnes and colleagues carefully evaluate the rigour of development of nine published guidelines using seven predefined criteria adapted from the Appraisal of Guidelines for Research and Evaluation (AGREE) framework. ${ }^{2}$ The authors conclude that the guidelines are inconsistent, and that the recommendations for "when to take action" are primarily based on consensus statements rather than scientific evidence.

Dinnes and colleagues highlight two reasons for the shortcomings they identified among the guidelines: inadequate use of available evidence and lack of data from appropriate studies in the literature. The inconsistencies in the specific recommendations among the nine guidelines were striking, and Dinnes and colleagues state "there does not appear to be any clear pattern in recommendations."2 This observation strongly suggests that most published guidelines have shortcomings that decrease their value for guiding clinical practice. In addition, Dinnes and colleagues sug- gest that this is likely true for guidelines in all areas of clinical medicine.

The guideline published by The National Institute for Health and Clinical Excellence (NICE) scored markedly better in the framework criteria than those issued by expert organizations. ${ }^{3}$ Indeed, it may come as no surprise that the institute, financed by the UK National Health Service, and specializing in providing guidelines for a wide range of disorders (with over 100 guidelines published to date), has developed rigorous methods. In particular, the guideline from NICE was the only one to consistently provide clear links between its specific recommendations and the underlying scientific evidence. However, it would appear that the apparent lack of formal scientific rigour among the other guidelines is a matter that could be easily remedied by collaborations between clinical experts and experts in the methodology of guideline development.

Although it is clear that existing evidence was not always optimally incorporated into the guidelines studied, there are limited data on the topic of monitoring patients with prostate cancer in the literature. This would seem a greater obstacle for the development of evidence-based guidelines than the lack of methodological rigour.

The gold standard study design in the evaluation of therapeutic strategies is the randomized controlled trial. However, comparing follow-up among men with localized prostate cancer is cumbersome because such cancers have a protracted clinical course that rarely leads to the patient's death. ${ }^{4}$ Furthermore, a large proportion of men with prostate cancer are older, and their risk of death from other causes is substantial. Hence, such studies are both costly and timeconsuming. Despite this lack of high-quality sci-

\section{KEY POINTS}

- The lack of involvement of methodologists in the development of guidelines may result in recommendations that do not reflect the evidence.

- Clinical experts should collaborate with methodologists on the design of clinical guidelines and the extraction of data.

- There is an urgent need for clinical trials in virtually all areas of the clinical management of prostate cancer. 
entific data, testing serum levels of prostatespecific antigen is frequently used for the early detection of prostate cancer, its surveillance and detection of relapse after curative treatment.

Although there are some ongoing prospective observational studies on monitoring strategies for the active surveillance of men with low risk for prostate cancer, ${ }^{5-7}$ to the best of our knowledge, there are no clinical trials on optimal monitoring strategies for men who have received curative treatment. In fact, it was only recently that trials showed reduced mortality from prostate cancer due to systematic testing of serum levels of prostatespecific antigen among healthy men, followed by curative treatment after diagnosis is confirmed. ${ }^{8,9}$

Thus, there is limited evidence to support even basic decisions on managing prostate cancer, and even less regarding the specific issue investigated by Dinnes and colleagues: optimal monitoring using prostate-specific antigen in men who have received curative treatment for localized prostate cancer. Indeed, it is doubtful if there will ever be a sufficiently large randomized trial of monitoring patients with prostate cancer after curative treatment. As such, the developers of guidelines will have to base their recommendations on other sources of information in the foreseeable future.

In the absence of data from randomized trials, some knowledge may be gleaned from highquality, population-based, cancer-specific registries. ${ }^{10,11}$ Although there are inherent pitfalls in the interpretation of observational studies, such as confounding by indication to treatment, if attention is paid to their design, these studies can provide valuable information (e.g., long-term outcomes when prostate cancer is treated using different strategies). ${ }^{12}$ Such data can subsequently be used in statistical modeling of the optimal use of prostate-specific antigen in surveillance, also accounting for competing risks.

The study by Dinnes and colleagues suggests that clinical experts would benefit from partnering with methodologists on the design of clinical guidelines and the extraction of data. Their survey highlights the urgent need for clinical trials in virtually all areas of clinical management of prostate cancer.

\section{References}

1. Dinnes J, Hewison J, Altman D, et al. The basis for monitoring strategies in clinical guidelines: a case study of prostate-specific antigen for monitoring in prostate cancer. CMAJ 2012;184:16977.

2. AGREE Collaboration. Development and validation of an international appraisal instrument for assessing the quality of clinical practice guidelines: the AGREE project. Qual Saf Health Care 2003; 12:18-23.

3. The National Institute for Health and Clinical Excellence. Prostate cancer: diagnosis and treatment. London (UK): The Institute; 2008.

4. Pound CR, Partin AW, Eisenberger MA, et al. Natural history of progression after PSA elevation following radical prostatectomy. JAMA 1999;281:1591-7.

5. Klotz L, Zhang L, Lam A, et al. Clinical results of long-term follow-up of a large, active surveillance cohort with localized prostate cancer. J Clin Oncol 2010;28:126-31.

6. van den Bergh RC, Roemeling S, Roobol MJ, et al. Prospective validation of active surveillance in prostate cancer: the PRIAS study. Eur Urol 2007;52:1560-3.

7. World Health Organization. A Swedish trial comparing two different follow-up schedules for patients with low-risk prostate cancer on active surveillance with selective, delayed treatment SAMS-FU. Geneva (Switzerland): The Organization; 2011. Available: http://apps.who.int/trialsearch/trial.aspx?trialid =ISRCTN64891728 (accessed 2011 Nov. 28).

8. Schröder FH, Hugosson J, Roobol MJ, et al. Screening and prostate-cancer mortality in a randomized European study. $N$ Engl J Med 2009;360:1320-8.

9. Hugosson J, Carlsson S, Aus G, et al. Mortality results from the Goteborg randomised population-based prostate-cancer screening trial. Lancet Oncol 2010;11:725-32.

10. Hagel E, Garmo H, Bill-Axelson A, et al. PCBaSe Sweden: a register-based resource for prostate cancer research. Scand J Urol Nephrol 2009;43:342-9.

11. Adolfsson J, Garmo H, Varenhorst E, et al. Clinical characteristics and primary treatment of prostate cancer in Sweden between 1996 and 2005. Scand J Urol Nephrol 2007;41:456-77.

12. Stattin P, Holmberg E, Johansson JE, et al. Outcomes in localized prostate cancer: National Prostate Cancer Register of Sweden follow-up study. J Natl Cancer Inst 2010;102:950-8.

Affiliations: Matthias Johansson is with the International Agency for Research on Cancer (Johansson), Lyon, France; Pär Stattin is with the Department of Surgery and Perioperative Sciences, Urology and Andrology, Umeå University Hospital, Umeå, Sweden, and the Department of Surgery, Urology Service, Memorial Sloan-Kettering Cancer Center, New York, NY.

Contributors: Mattias Johansson and Pär Stattin both contributed intellectually to this work, drafted the manuscript and approved the final version for publication. 